\title{
El reto de la calidad para el Tercer Sector Social. Análisis de casos de implantación del modelo EFQM
}

\author{
The challenge of quality for Social Third Sector. \\ Cases analysis of EFQM model implementation
}

\author{
Emma JuAneda Ayensa ${ }^{1}$ \\ LeONor GonZÁlez Menorca ${ }^{1}$ \\ Carmen Marcuello Servós ${ }^{2}$ \\ Universidad de La Rioja (España) \\ Universidad de Zaragoza (España)
}

Recibido el 3 de mayo de 2011, aceptado el 8 de febrero de 2013

$\mathrm{N}^{\mathrm{o}}$ de clasificación: L31 y M11

DOI: $10.5295 / \mathrm{cdg} .110285 \mathrm{ea}$

\section{Resumen:}

Las dificultades de los gobiernos occidentales para mantener los Sistemas de Bienestar han promovido la privatización en la gestión de los servicios. Las organizaciones del Tercer Sector, en su papel como proveedoras de servicios, deben adaptarse a los cambios provocados por las demandas sociales y tratar de fomentar su participación en la configuración de los sistemas, pero siendo capaces de demostrar la eficiencia con la que desarrollan sus actividades. Ante este reto, los sistemas de gestión de la calidad ofrecen una oportunidad a las organizaciones del Tercer Sector, aunque por parte de las entidades que conforman el sector se mantienen ciertas reticencias sobre la validez del modelo a causa de su origen empresarial.

Este trabajo de investigación tiene por objetivo el estudio de los procesos de implantación de un sistema de Gestión de la Calidad en las organizaciones del Tercer Sector Social. Para ello se llevó a cabo un análisis de casos múltiples, eligiendo cuatro organizaciones en diferentes estadios de implantación. El tratamiento de la información se realizó mediante el software de análisis cualitativo Atlas.ti y, para la evaluación de cada caso, se siguió el sistema de valoración del EFQM, analizando los diferentes elementos de los agentes facilitadores. Los principales resultados muestran la importancia de las alianzas, el enfoque en el usuario así como en las personas, los cuales son considerados como elementos clave para estas organizaciones. El trabajo muestra las conclusiones para cuatro casos, pero sugiere la importancia de establecer las relaciones entre los agentes facilitadores así como un análisis causal para futuras líneas de investigación.

Palabras clave:

Sistemas de Gestión de Calidad Total, Modelo EFQM, Tercer Sector, Servicios Sociales, Análisis de casos.

\footnotetext{
${ }^{1}$ Universidad de La Rioja, Departamento de Economía y Empresa, c/ La Cigüeña, 60, 26004 Logroño (España), emma.juaneda@unirioja.es, leonor.gonzalez@unirioja.es

${ }^{2}$ Universidad de Zaragoza, Departamento de Economía y Dirección de Empresas, c/ Gran Via, 2, 50005 Zaragoza (España),cmarcue@unizar.es
} 


\begin{abstract}
:
Nowadays, the difficulties of the Governments to maintain the Welfare Systems have promoted the private management in the welfare services sector. The Third Sector organizations, as welfare services suppliers, have to adapt to the changing social demands and try to encourage their participation in the system configuration but being able to demonstrate the efficiency with which they operate. Faced with this challenge, Quality Management Systems offer an opportunity for the Third Sector Organizations, although with some reluctance due to the industrial origin of the model.

The main aim of this work is to study the implementation process of a Total Quality Management system in the Third Social Sector organizations. Our research has been done through the analysis of multiple cases, selecting four organizations in different stages of quality management implementation. The data analysis was done using qualitative analysis software ATLAS.ti and for the evaluation of each case was done following the EFQM assessment system software to analyze the different elements of the model enablers. The main results are the relevant meaning of alliance development, the user focus as well as the person focus, elements system which the organizations consider as key issues. This research work shows the process for four organizations but suggest the necessity to research deeply about relationship among the enablers as well as a causal analysis as future lines.
\end{abstract}

\title{
Keywords:
}

Total Quality Management Systems, EFQM Model, Third Sector Organizations, Social Services; Cases Analysis. 


\section{INTRODUCCIÓN}

En la década de los ochenta, la crisis de los Estados de Bienestar puso de manifiesto las dificultades de los gobiernos para el sostenimiento perdurable de los compromisos sociales adquiridos. Este hecho supuso la incorporación al sistema de otros "agentes", pasando de un Welfare State al actualmente denominado Welfare-Mix, convertido en un partenariado entre el Estado y las organizaciones privadas: no lucrativas y empresas. En este contexto, el Tercer Sector Social ha evolucionado: de entidades creadas bajo el auspicio de la beneficencia de sus fundadores han pasado a ser entidades proveedoras de servicios de interés general, receptoras de fondos públicos y con un tratamiento fiscal preferente debido a su naturaleza social. Por ello, ya no es suficiente la buena voluntad que impulsó su nacimiento, sino que deben trabajar de manera eficiente y además, deben ser capaces de demostrar la calidad de su trabajo.

Los sistemas de Gestión de la Calidad se han vinculado tradicionalmente al ámbito industrial por ser el sector en el que se realizaron las primeras aportaciones. Desde sus orígenes hasta su actual conceptualización, los sistemas de Gestión de la Calidad Total o Excelencia (TQM) han ampliado su significado hasta lograr convertirse en sistemas de gestión holísticos que favorecen la mejora continua, la orientación al cliente y la visión global de la organización, promoviendo a su vez la adaptación constante al entorno y a las expectativas de los distintos grupos de interés (Juaneda Ayensa 2010, p.148). Aunque los principios puedan ser considerados universales, su extensión a otros sectores ha requerido adaptaciones previas, y es que la concreción de los modelos y prácticas ha de realizarse adecuándolos a las circunstancias específicas (Shin et al. 1998). En este proceso expansivo, en el que los servicios de naturaleza pública tardaron en incorporarse, fue el sector sanitario el que inició este camino hacia la Excelencia, seguido por el de la educación y, posteriormente, por el sector de servicios sociales.

El Tercer Sector, especialmente el que trabaja en el ámbito de los servicios sociales, comúnmente denominado Tercer Sector Social (TSS), presenta ciertas peculiaridades que condicionan su funcionamiento (Herrera Gómez et al. 2000). Algunas de ellas ventajosas como por ejemplo la ausencia de intereses privados que le confiere su naturaleza "no lucrativa", o su capacidad de actuación como instrumentos de democratización social; pero también hay inconvenientes, como la ausencia de derechos de propiedad -lo que no incentiva a la dirección de estas organizaciones de manera eficiente-, el escaso control sobre el desempeño, o el alto nivel de dependencia de los fondos públicos, entre otros (Sajardo y Chaves 2006). Esta elevada dependencia del sector público conlleva que las decisiones estratégicas tomadas por las organizaciones del sector sean más sensibles a los cambios de las políticas gubernamentales y a los consiguientes recortes presupuestarios. Otra característica a resaltar es la existencia de un complejo mapa de stakeholders, los cuales ejercen una influencia considerable tanto en el sistema operativo como en su legitimización. En definitiva, la naturaleza social del sector se conjuga con su fuerte identidad, producto de un complejo conjunto de factores históricos (Salamon y Anheier 1998, p.245) y de nuevos retos, lo que exige a las organizaciones del TSS un constante replanteamiento de medios y de resultados.

Así pues, considerando estas características y contemplando los requisitos para la implantación de la TQM (Hamlin et al. 1997; Zeitz et al. 1997; Abraham et al. 1999; Ade- 
banjo y Kehoe 1999; Ho et al. 1999; Aguirre García y Aparicio de Castro 2002; Camisón et al. 2007; Martínez Caro et al. 2010)ํㅜ, nuestro propósito es el estudio de los procesos de implantación y de los principales cambios a los que se enfrentan las OTSS durante el proceso. El objetivo es de carácter constructivista, siguiendo un enfoque deductivo desde el que se pretende aplicar un marco general (Sistemas TQM) a un contexto particular (TSS) basándolo en la información procedente de casos de organizaciones que han sido pioneras en la implantación de un sistema TQM basado en el modelo EFQM. Así pues, presentamos en el primer apartado un recorrido por las principales iniciativas desarrolladas por el Tercer Sector para adaptar a su realidad la calidad en la gestión. Posteriormente, en el epígrafe dedicado a la metodología, se argumentan las razones de la elección realizada y se presenta el diseño de la investigación realizada. A continuación, se exponen los principales resultados en relación con las actuaciones llevadas a cabo en los diferentes agentes facilitadores, así como la descripción del contexto en el que se ha realizado la implantación. Finalmente, en el último apartado, se presentan las conclusiones y las futuras líneas de investigación.

\section{LA CALIDAD COMO UNA OPORTUNIDAD PARA EL TERCER SECTOR}

Durante la última década, el Tercer Sector, consciente de la necesidad de justificar su funcionamiento e introducir mejoras en él, ha desarrollado diferentes propuestas para promover la Gestión de la Calidad, algunas de ellas originales y otras basadas en modelos generalistas pero adaptados a sus peculiaridades. A raíz de estas experiencias pioneras, se han venido impulsando dos grandes líneas de actuación en España: la dirigida a crear un sistema de calidad propio del sector, la llamada ONGconcalidad, y la promoción de la implantación de un sistema de gestión basado en el modelo EFQM.

El sistema ONGconcalidad es una propuesta impulsada por FEAPS, Secretariado General Gitano, Cruz Roja, Fundación Vínculos, Federación de Mujeres Progresistas, Fundación Pioneros e INTRESS. Al igual que la norma ISO 9001, es un sistema para la certificación que garantiza un nivel de gestión. El sistema ha sido experimentando en servicios de las citadas ONG, intentando configurarse como una Red de Cooperación para la TQM. En el marco de esta iniciativa, el 15 de abril de 2008 se creó el Instituto para la Calidad en las ONG (ICONG) en el que se integran las entidades de la Red ONGconcalidad. Actualmente se encuentran en la tercera versión de la norma, que fue publicada en el año 2009.

De igual forma, debe destacarse el trabajo realizado por la Fundación Luis Vives por encargo del Ministerio de Trabajo y Asuntos Sociales, consistente en analizar modelos internacionales de gestión de calidad en servicios sociales, especialmente el modelo EFQM y observar sus cualidades y características para ser aplicadas en el contexto de las ONG. A raíz de este estudio, esta fundación desarrolló un programa de ayuda para la implantación del modelo EFQM en el TSS que fue financiado por el Fondo Social Europeo.

\footnotetext{
${ }^{1}$ Véase Juaneda (2010, p.166 y 186) para una revisión más amplia de los trabajos en los que se analiza la implantación de los sistemas de calidad y los sus resultados.
} 
Otro proyecto más reciente es el sistema online de indicadores compartidos para la mejora de la calidad en la acción social. Esta iniciativa, impulsada por la Red de Consultoría Social integrada por INTRESS, Fundación Pere Tarrés, Fundación EDE, CIREM y SUSTENTIA, ha sido financiada por el Ministerio de Sanidad y Política Social. Como resultado, se elabora una Guía de indicadores que permitan medir los resultados de la acción social agrupados en seis categorías de Indicadores: claves no económicos, claves económicos, de gestión, de Destinatarios-personas, de Destinatarios-financiadores, y del equipo de personas (Sánchez Aguado 2011).

Vinculadas a la mejora aunque en distinta línea, han aparecido propuestas como la de Fundación Lealtad que plantea la evaluación de las organizaciones en relación a su grado de transparencia, argumentando que los resultados inciden sobre la economía, gobierno y transparencia de las ONG y pueden contribuir indirectamente a una mejora global de la gestión. Desde que se creó en el año 2001, se han editado seis guías de evaluación de las buenas prácticas y la transparencia de las ONGs.stas han aparecido otras i

Otra iniciativa pionera es el modelo de calidad FEAPS, desarrollado entre los años 2004 y 2006. Se trata de un modelo específico para organizaciones dedicadas a la discapacidad intelectual y lo destacamos aquí por lo que supone: la ampliación del concepto del modelo de calidad. La calidad en la gestión es definida por la entidad como el resultado de la interacción de tres componentes esenciales: calidad de vida, calidad en la gestión y ética.

También hay que resaltar la realización de una Norma UNE de Gestión Ética de las ONG por parte de AENOR; además, el modelo EFQM tiene una versión adaptada al Sector Público y Entidades del Voluntariado (EFQM 2003).

Todas estas propuestas están siendo desarrolladas, lo que permite prever que en los próximos años se logrará una mayor claridad y eficacia a la hora de adoptar alguno de los sistemas, lo que probablemente será obligatorio para aquellas organizaciones que quieran optar a la financiación pública y captación de fondos privados.

\section{METODOLOGÍA}

En este trabajo se pretende dar respuesta a cómo se realiza el proceso de implantación del modelo EFQM en entidades sociales y los elementos principales que lo condicionan, por ello, los objetivos generales del trabajo se desglosaron en las preguntas de la investigación que se recogen en el Anexo 1.

Para alcanzar los objetivos propuestos en este trabajo se optó por una metodología cualitativa que nos permitiera aproximarnos in situ al contexto del TSS ante el desafío de la implantación de un sistema TQM. Los sistemas TQM implantados en organizaciones del TSS son un fenómeno incipiente que se encuentra en desarrollo y por ende, existe poca investigación sobre el tema. Aunque el uso de los métodos cualitativos no ha sido habitual en disciplinas como la Economía de la Empresa, su utilidad comienza a ser valorada cuando se analizan situaciones en las que se conoce poco sobre un fenómeno dado, o se trata de estadios iniciales de la investigación sobre una nueva área temática; también demuestran su eficacia en el análisis de procesos de cambio longitudinales, o bien cuando se trata de ámbitos donde las perspectivas teóricas existentes parecen ser inadecuadas o tienen una escasa sustentación empírica (Eisenhardt 1989). 
En este trabajo, nuestro objetivo no es contrastar hipótesis generales en un contexto particular, sino descubrir las diferentes maneras en que estas organizaciones particulares llevan a cabo un proceso de implantación de un sistema de gestión de calidad -recuérdese que se trata de un instrumento originariamente procedente del ámbito empresarial- en organizaciones que no tienen fines lucrativos y son de naturaleza social. Por otra parte se pretende, desde una perspectiva práctica, dar a conocer los resultados de experiencias pioneras en el TSS y la utilidad de los sistemas TQM para la mejora de su organización, y con ello, facilitar el logro de sus fines.

Planteados los objetivos y decidida la metodología, se diseñó una estrategia de investigación para tratar de garantizar la objetividad y validez de la información para la cual se siguieron las recomendaciones de Yin $(1994,1998)$ que quedan reflejadas de manera sintética en la siguiente Tabla 1.

Tabla 1

\section{Validez y Fiabilidad}

\begin{tabular}{ll}
\hline Validez Conceptual & $\begin{array}{l}\text { Utilización de diversas fuentes de información: documentación } \\
\text { proporcionada por las organizaciones, fuentes secundarias y entrevista. }\end{array}$ \\
\hline Validez Interna & $\begin{array}{l}\text { Triangulación en el análisis de la información. } \\
\text { Creación de redes relacionales replicante entre los documentos para } \\
\text { la interpretación conjunta de la información procedente de varias } \\
\text { organizaciones. }\end{array}$ \\
\hline Validez Externa & $\begin{array}{l}\text { Aplicación de la lógica replicante de los casos múltiples mediante } \\
\text { heterogeneidad de la organizaciones y diferencias en los estadios de }\end{array}$ \\
& implantación. \\
\hline Fiabilidad & $\begin{array}{l}\text { Diseño de Protocolo del estudio. } \\
\text { Elaboración de una guía de la entrevista estructurada basada en los } \\
\text { diferentes modelos de TQM y los cuestionarios de trabajos previos (Caro, }\end{array}$ \\
& $\begin{array}{l}\text { 2001; Grandzol y Gershon, 1998; Lorenzo, 2003; Aguirre García et al. } \\
\text { 2003). }\end{array}$ \\
& Realización de un Caso piloto \\
\hline
\end{tabular}

El diseño de la guía de la entrevista se realizó considerando los elementos de los modelos EFQM para el Sector Público y las entidades de Voluntariado (EFQM, 2003) y otros trabajos previos (Granzol y Gershon 1998; Caro 2001; Aguirre et al. 2003; Lorenzo 2003). El objetivo era obtener información referente a dos bloques: los factores que condicionan el cambio (motivaciones internas, presiones externas, frenos a la implantación y cambios organizativos recientes) y los elementos para la medición de la TQM (agentes facilitadores del modelo EFQM). El proceso definido para la recogida y el análisis de la información se presenta de forma esquemática en la Figura 1. 
Figura 1

Desarrollo del método del caso

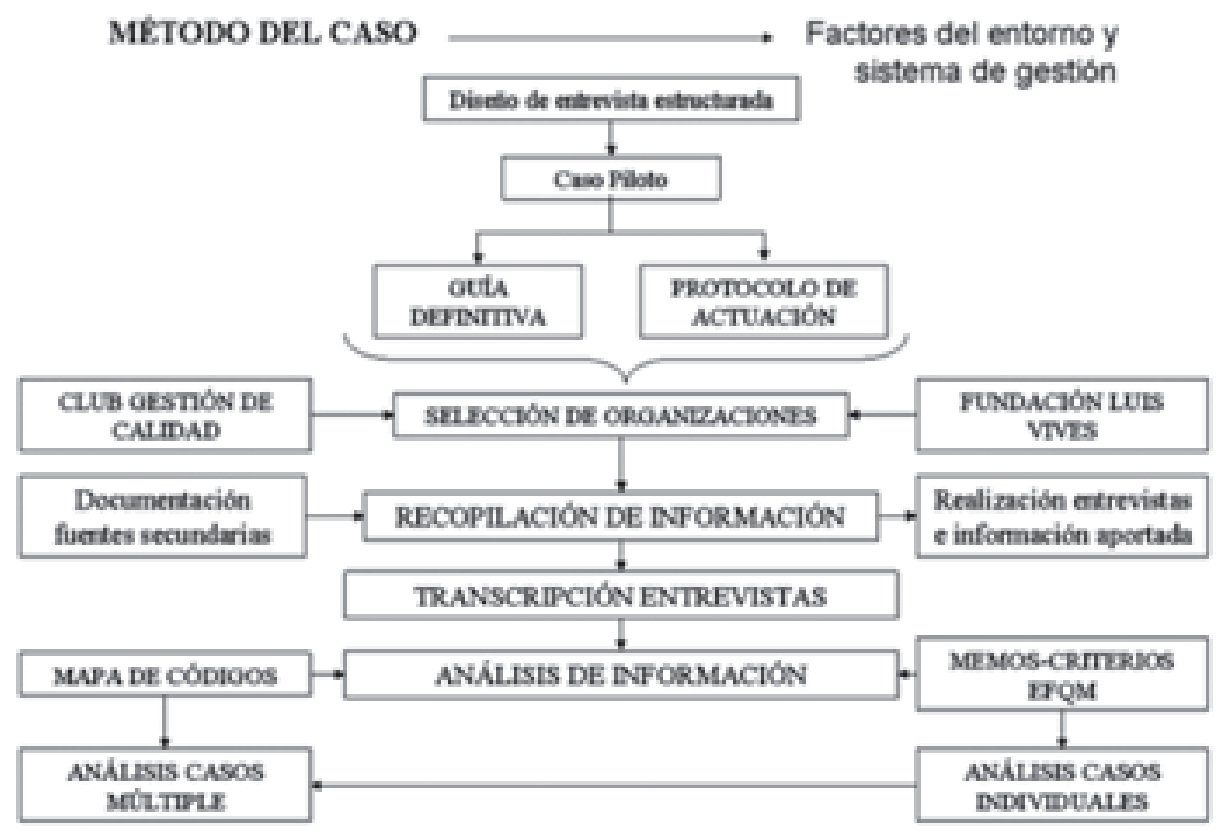

Posteriormente, se procedió a elegir las organizaciones. Los primeros requisitos fueron, obviamente, que hubieran implantado un sistema de gestión de calidad basado en el modelo EFQM y que prestaran servicios de atención directa en el sector de servicios sociales. Se plantearon varias alternativas en función del tiempo que llevasen implantando el modelo (de un año a cuatro años) y se hizo una preselección de ocho entidades ${ }^{2}$. Los criterios fueron: forma jurídica, principales beneficiarios, año de implantación, e interés y preocupación por la mejora para tratar de asegurar que existiese una predisposición a la colaboración. Tras una primera toma de contacto con las organizaciones y considerando su predisposición para colaborar, se realizó el análisis de casos final con cuatro de ellas.

El tratamiento de la información se llevó a cabo mediante el software de análisis cualitativo Atlas.ti.y la valoración de las organizaciones, mediante la herramienta de autoevaluación PERFIL basada en el modelo EFQM. La toma de datos se realizó en la primavera de 2007.

\footnotetext{
${ }^{2}$ Debido a la ausencia de información sobre las organizaciones que tuvieran implantado un sistema TQM basado en el EFQM y que hubieran obtenido un Sello de Calidad, nos pusimos en contacto con la Fundación Luis Vives que llevaba un programa de asesoramiento para la implantación en entidades de Acción Social. Por tanto, siete de las ocho entidades formaban parte del programa de la fundación.
} 


\section{RESULTADOS DEL ANÁLISIS DE CASOS MÚLTIPLE}

Las entidades elegidas son dos fundaciones y dos asociaciones declaradas de interés general. En la Tabla 2 se presentan esquemáticamente las características principales de las organizaciones.

Tabla 2

\section{Caso Múltiple}

\begin{tabular}{|c|c|c|c|c|}
\hline CASO & $\mathrm{A}$ & B & $\mathrm{C}$ & $\mathrm{D}$ \\
\hline Forma jurídica & Fundación & Asociación & Fundación & Asociación \\
\hline Área actuación & Regional & Local & Nacional & Regional \\
\hline Tipo & Interés General & Ayuda Mutua & Interés General & Ayuda Mutua \\
\hline $\begin{array}{l}\text { Principales } \\
\text { Beneficiarios }\end{array}$ & $\begin{array}{l}\text { Colectivos en } \\
\text { situación o } \\
\text { en riesgo de } \\
\text { exclusión } \\
\end{array}$ & $\begin{array}{l}\text { Personas con } \\
\text { discapacidad } \\
\text { física }\end{array}$ & Infancia & $\begin{array}{l}\text { Personas } \\
\text { dependientes y a } \\
\text { sus familiares }\end{array}$ \\
\hline $\begin{array}{l}\text { Categoría } \\
\text { Dominante }\end{array}$ & $\begin{array}{l}\text { Patronato y } \\
\text { Asamblea }\end{array}$ & $\begin{array}{l}\text { Junta directiva- } \\
\text { socios: Enfermos }\end{array}$ & Patronato & $\begin{array}{l}\text { Junta directiva- } \\
\text { socios: Familiares }\end{array}$ \\
\hline Origen & 1981 & 1991 & 2000 & 1993 \\
\hline $\begin{array}{l}\text { Comienzo } \\
\text { Implantación }\end{array}$ & 2002 & 2004 & 2006 & 2005 \\
\hline Motor & $\begin{array}{l}\text { Motivación } \\
\text { Propia: Mesa de } \\
\text { Dirección } \\
\text { Mejora interna. }\end{array}$ & $\begin{array}{l}\text { Junta directiva y } \\
\text { Dirección. } \\
\text { Mejora interna. } \\
\text { Reconocen la } \\
\text { posibilidad de } \\
\text { ser exigido en el } \\
\text { futuro. }\end{array}$ & \begin{tabular}{|l|} 
Dirección. \\
Visión objetiva \\
de la calidad del \\
trabajo realizado. \\
Lograr un aval \\
que facilitara \\
la obtención de \\
financiación. \\
Fundación Matriz \\
\end{tabular} & $\begin{array}{l}\text { Confederación } \\
\text { Nacional } \\
\text { Percepción de } \\
\text { requisito por las } \\
\text { Administraciones } \\
\text { Públicas a raíz } \\
\text { de la ley de } \\
\text { Dependencia. }\end{array}$ \\
\hline Apoyo Inicial & $\begin{array}{l}\text { Entidad de pro- } \\
\text { moción regional. } \\
\text { Posteriormente } \\
\text { supervisado por } \\
\text { la Fundación Luís } \\
\text { Vives }\end{array}$ & $\begin{array}{l}\text { Fundación Luís } \\
\text { Vives }\end{array}$ & $\begin{array}{l}\text { Fundación Luis } \\
\text { Vives. } \\
\text { Fundación Matriz. }\end{array}$ & Consultor externo \\
\hline Freno Principal & $\begin{array}{l}\text { Falta de recursos } \\
\text { específicos-No } \\
\text { vinculado con la } \\
\text { Misión }\end{array}$ & $\begin{array}{l}\text { Falta de recursos } \\
\text { adicionales-queda } \\
\text { relegado por la } \\
\text { actividad propia } \\
\text { de la asociación. } \\
\text { Origen y } \\
\text { orientación } \\
\text { empresarial de los } \\
\text { modelos. }\end{array}$ & $\begin{array}{l}\text { Carencia } \\
\text { de personal } \\
\text { disponible. }\end{array}$ & $\begin{array}{l}\text { Falta de } \\
\text { adaptación del } \\
\text { Modelo al ámbito } \\
\text { social. }\end{array}$ \\
\hline
\end{tabular}


La constitución de las organizaciones es reciente -tres son de los años noventa-; una de ellas se constituyó en 1981 y es la que tiene más experiencia en el desarrollo del sistema basado en el EFQM.

La precariedad de recursos en sus inicios es una característica común, hasta que la consolidación de la organización les permitió mejorar los medios para acceder a las ayudas públicas. Sin embargo, todas muestran un deseo de independencia financiera, pues son conscientes de que, con frecuencia, sus programaciones se ven afectadas por intereses políticos.

Las organizaciones de dimensión local-regional se encuentran integradas en organizaciones de nivel nacional o supranacional que tienen los mismos fines sociales; así, las dos entidades de familiares de enfermos están amparadas por una federación o confederación nacional, o participan en diversas redes con objetivos y misiones similares a la suya.

Conscientes de que la implantación de sistemas de calidad es muy beneficiosa para el desarrollo de las entidades, la iniciativa de implantarlos parte de las mismas entidades, aunque reconocen el impulso externo positivo que reciben de programas de fomento de la calidad desarrollados por instituciones públicas, o por las confederaciones nacionales; destacan la ayuda ofrecida por la fundación Luis Vives para las fases previas.

Los problemas que hemos detectado en la implantación, comunes a todas las entidades, son:

a) Dificultad de manejar un lenguaje que está orientado a objetivos empresariales, tanto por la terminología específica como por los propios objetivos a conseguir.

b) Dificultad de afrontar los costes económicos de la implantación, no tanto por el volumen total, que a veces no representa un alto porcentaje sobre el presupuesto, sino porque las entidades son reacias a desviar dinero de sus objetivos, lo que dificulta el poder afrontar los costes de tiempo empleado en la implantación.

c) A ello debemos añadir la dificultad de obtener financiación del sistema de calidad a través de los cauces normales debido a que no se considera un objetivo enlazado con la misión social propia de la entidad

A continuación analizaremos las diferentes fórmulas utilizadas en cada uno de los agentes facilitadores del modelo EFQM.

\subsection{Liderazgo}

Uno de los elementos desencadenante del desarrollo del proceso es el Liderazgo. El análisis del proceso de implantación muestra cómo el equipo técnico es el encargado de realizar las funciones de liderazgo. El Liderazgo que promueve la implantación una vez tomada la decisión es el equipo técnico, frente al componente voluntario (Junta Directiva/ Patronato) que es el que aprueba la decisión. Esto se debe a que son los principales responsables de la función operativa, aunque las decisiones estratégicas son tomadas por el Patronato/Junta Directiva.

En todas las organizaciones existe una trayectoria previa con una marcada orientación de sus actividades hacia la Misión, establecida por los propios estatutos, y que condiciona la definición de la Visión y los Valores que se realiza en las fases previas de implantación. Así como los Valores son implícitos a la propia organización -aunque es en el proceso de implantación cuando se "materializan"-, sin embargo, la definición de Visión no es tan evidente y requiere una orientación de futuro que no se ha realizado de manera informal. Por 
otra parte, las organizaciones llevan a cabo actuaciones para publicitar su Misión, siendo especialmente difundida por la Fundación C, que hace especial incidencia sobre ella en todos los documentos públicos de la entidad, siendo esta organización una entidad con un porcentaje financiación privada y autofinanciación elevado.

La implicación en el proceso de revisión se ha configurado sólidamente en el Caso A, entidad con mayor experiencia en el modelo, mientras que en las restantes organizaciones el proceso de revisión está definido en menor grado, aunque se realizan revisiones de los planes estratégicos.

El compromiso con la calidad se ve reflejado a través de la participación en los cursos de formación, la incorporación de la dirección en los comités de calidad y la incorporación de la calidad en el plan estratégico.

\subsection{Política y Estrategia}

Las organizaciones han desarrollado sus planes estratégicos integrando la calidad como objetivo estratégico en el diseño de los Planes, de tal manera que pueda promoverse a través de ellos el logro de los principios que promueve la TQM.

Aunque todas ellas hayan adoptado alguno de los objetivos de mejora como elementos de los planes definidos, comprobamos que el nivel de despliegue así como de incorporación de las expectativas de los diferentes grupos de interés no son equiparables entre las diferentes organizaciones. En el proceso de definición, la Fundación A incorpora de diversas maneras a todos los miembros de la organización, desarrolla los planes, los implanta y revisa. En los casos B y D, la participación interna de la organización no se ha instrumentalizado directamente, pero debemos destacar la orientación al usuario, promovida desde la naturaleza asociativa. En estos casos, la definición de los planes es realizada por los propios beneficiarios, por tanto, la información sobre sus necesidades y demandas se incorpora de manera directa. Las organizaciones desarrollan la participación en redes sociales como instrumentos para obtener información del entorno, aunque el uso de herramientas como el Benchmarking se realiza de forma muy limitada.

\subsection{Personas}

La orientación a las personas es uno de los pilares en los que se sustenta la TQM y uno de los elementos destacados por todas las organizaciones. El argumento principal es la consideración integral del usuario, es decir, la complejidad de las situaciones que requiere una intervención multidisciplinar para la provisión de un servicio global. Esta peculiaridad y el hecho de ser organizaciones proveedoras de servicios de atención directa confieren a los recursos humanos un papel relevante. El desarrollo de la misión y los objetivos de la organización está asociada con la definición de usuario y del servicio de intervención, elementos clave que condicionan la estructura de las organizaciones. Así pues, podemos observar que las organizaciones que desarrollan una definición compleja del servicio favorecen el trabajo en equipo mediante la provisión a través de actuaciones multidisciplinares y coordinadas. 
La formación de los recursos humanos ha sido identificada como un componente crítico de la Dirección de RRHH cuando se van a implementar importantes cambios organizativos, como es el caso de la aplicación de un sistema TQM.

En general, destacaremos algunos principios a tener en cuenta en el proceso:

1. El trabajo en equipo y la cooperación entre los miembros deben ser fomentadas con el fin de adaptarse a las necesidades de los usuarios ante demandas complejas que requieren intervenciones multilaterales.

2. La planificación y mejora de las competencias de los RRHH incorporan como elementos fundamentales los valores. Las competencias deben estar definidas en sintonía con la misión, y alcanzadas por medio de la contratación y la formación.

3. La importancia de un sistema de comunicación interna que permita obtener información para la coordinación de y entre los equipos así como la información desde la base para la toma de decisiones estratégicas.

4. Los sistemas de reconocimiento que incorporen recompensas, aunque no se señalan como relevantes las de carácter económico.

En general, las cuatro organizaciones coinciden en la existencia un alto nivel de implicación y por tanto, su trabajo se realiza en un ambiente grato, en el que a pesar de los salarios bajos -el gran problema detectado- prima la cooperación y, por encima de todo, la atención a los usuarios.

\subsection{Alianzas y Recursos}

Según el modelo enfocado hacia la empresa, la peculiaridad de las alianzas va dirigida a integrar los procesos externos de las organizaciones que les proporcionan bienes o servicios. Una de las características reseñables de los modelos es que el concepto de cooperación se amplía no sólo a la cooperación con las organizaciones que forman la cadena de valor, sino que la cooperación es horizontal.

Este principio implica la búsqueda de las alianzas con entidades u organismos que tengan establecida una misma o similar misión a la organización. Esta característica puede ser entendida debido a su misión vinculada con un beneficio general. La misión asociada a un servicio social implica que no es la organización la que exclusivamente deba procurar que la misión sea alcanzada sino que, si no es por ella, lo sea por otras organizaciones o a través de redes: cooperación versus competencia. Esta actitud es la que favorece el desarrollo de alianzas con otro tipo de entidades. Las alianzas pueden ser de distinta naturaleza dependiendo del fin que le otorgue la organización (captación de fondos, realización de proyectos conjuntos, o actuaciones reivindicativas).

La implantación de este principio se lleva a cabo a través de prácticas como:

1. Establecimiento de alianzas estratégicas o de programas de cooperación con sus financiadores integrándolos como proveedores. La reducción de la base de financiadores privados busca una identificación entre proyecto-entidad financiadora y puede redundar en la existencia de relaciones más estrechas y continuas en el tiempo.

2. La cooperación con otras entidades mediante equipos conjuntos y redes les permite participar en el diseño de acciones colectivas, así como actuar como lobby de poder que canalice las demandas sociales, sus quejas y sugerencias de mejora a los poderes públicos. 
3. La integración en otra entidad suprarregional o redes de trabajo permiten el desarrollo de programas de mejora. Estos programas facilitan el intercambio de conocimiento, información, experiencias o desarrollar nuevas competencias, para que aprendan a gestionar y mejorar la calidad y la eficiencia de sus proyectos desde un enfoque social.

\subsection{Procesos}

El enfoque basado en procesos facilita la adopción de la orientación al usuario y la incorporación de la cultura de la mejora continua. Las medidas tomadas están encaminadas a:

1. Sustentar altos grados de participación con un clima organizativo de confianza, y cuyo diseño incorpore explícitamente criterios de reconocimiento a los resultados colectivos.

2. Romper las barreras horizontales y verticales, así como entre especialidades, que dificultan la realización del trabajo con satisfacción.

3. Desarrollar de relaciones laterales así como pautas de entrenamiento y aprendizaje interfuncionales.

La Fundación A es la que muestra una mayor interiorización del mapa de procesos y aplica una revisión regularmente, lo que responde seguramente a que su sistema de participación es muy desarrollado y a que es la entidad que tiene más experiencia con el modelo EFQM.

En general, la implantación ha sido realizada por las entidades con cierto éxito y satisfacción por parte de las mismas, aunque la dificultad se manifiesta en la interiorización y la revisión del propio sistema, pues éste es uno de los elementos que requiere mayor esfuerzo para su implementación completa y el logro de la mejora continua.

\section{CONCLUSIONES}

Como conclusión de carácter general podemos establecer que las entidades del TSS pueden obtener beneficios para su organización y mejorar su eficacia implantando sistemas de calidad. A pesar de la aparente contradicción entre su finalidad altruista y los sistemas de neto carácter empresarial, nuestro trabajo con cuatro organizaciones representativas demuestra no solo que las que han implantado el sistema han comprobado sus beneficiosas consecuencias, sino que además se encuentran en mejor disposición para responder en su momento ante los poderes públicos, las entidades patrocinadoras y los propios usuarios.

En referencia a los motivos que han promovido la decisión de adoptar el sistema TQM basado en el modelo EFQM, hemos comprobado que la decisión de la implantación surge de la iniciativa de la propia entidad, bien por una decisión de la directiva, o bien por el influjo de las confederaciones a las que pertenecen. Por los casos analizados podemos observar que las entidades que dependen en mayor medida de financiación externa privada consideran el modelo como un aval de la gestión realizada, mientras que las que mantienen un mayor enfoque en el usuario lo consideran como un instrumento para la autoevaluación.

En relación a la implantación del sistema, consideramos que las organizaciones del TSS cuentan con ciertos factores que favorecen la implantación: el trabajo cooperativo y desarrollo de alianzas, la implicación de las personas y la orientación al usuario. 
El desarrollo de alianzas surge en dos direcciones: horizontal e "integración” vertical. En el primero de los casos, las organizaciones buscan la cooperación con otras entidades con una misión similar para favorecer el logro conjunto. En el segundo de los casos, la "integración" es definida como el desarrollo de alianzas con entidades financiadoras que proporcionen fondos para el logro de la misión.

Las entidades manifiestan la existencia de un alto nivel de compromiso de sus miembros, que incluso se prolonga después de la finalización de las causas de establecimiento de la relación como usuarios, y que puede también manifestarse en los trabajadores de atención directa a los usuarios.

Finalmente, se aprecia la preocupación por la satisfacción de los usuarios como una premisa constante de funcionamiento. La misión y el fin último de su existencia es el usuario, por ello hallamos presente en diferentes grados la participación de los beneficiarios/ usuarios en la toma de decisiones organizativas, destacando las de carácter asociativo.

En la mayoría de los casos hemos observado que la gestión por procesos, como una revisión sistematizada y global de la actividad, es el agente facilitador menos desarrollado por las organizaciones. El primer paso, la elaboración del mapa de procesos, se ha desarrollado, aunque para las organizaciones resulta difícil su interiorización.

En definitiva, la implantación de sistemas TQM en el Tercer Sector Social va en aumento cada día. En general, las entidades que han implantado el sistema basado en el modelo EFQM se muestran satisfechas, aunque reconocen que muchos de los resultados no les sorprendían, pues venían "trabajando con calidad sin darse cuenta". Aunque el grado de satisfacción es alto desde la perspectiva de la Dirección, tras las evaluaciones iniciales se observa una especie de decadencia en el seguimiento posterior y una disminución del impulso hasta que no comienzan a materializarse los resultados de una manera evidente.

En adelante, habrá que seguir insistiendo en la necesidad de realizar un trabajo amplio sobre la gestión en las organizaciones del TSS y particularmente sobre la Gestión de la Calidad. En este sentido, nuestro trabajo debiera contribuir con una ampliación en dos direcciones. Por una parte, como un sistema de calidad es una apuesta a medio-largo plazo, resulta de gran interés analizar los resultados obtenidos después de años de implantación, especialmente los relativos a la evolución de los resultados sobre los grupos de interés y sobre los usuarios de servicios, así como las percepciones de estos grupos. Por otra parte, al haberse difundido la calidad en el sector y por tanto, haber aumentado el universo poblacional, un estudio con una muestra mayor nos permitiría incorporar nuevas variables en las relaciones entre los diferentes factores del modelo EFQM.

\section{BIBLIOGRAFÍA}

Abraham M., Crawford J. and Fisher T., 1999. Key factors predicting effectiveness of cultural change and improved productivity in implementing TQM. Journal of Quality and Reliability Management, 6 (2), 112-132.

Adebanjo D. and Kehoe D., 1999. An investigation of quality culture development in UK industry. International Journal of Quality and Reliability Management, 19 (7), 633-649.

Aguirre Garcia, M.S. and Aparicio de Castro M.G., 2002. La gestión de calidad y el marketing interno como factores de competitividad en empresas de servicios: El caso de empresas vascas de servicios con gestión avanzada. Cuadernos de Gestión, 2(2), 27-49. 
Aguirre García M.S., Albizu Gallastegui E., Charterina Abando J., Forcada Sainz F.J. and Landeta Rodríguez J., 2003. Análisis de la calidad en la gestión de las PYMES de la CAPV y de su incidencia en los resultados económicos. Proyecto UPV/EHU y CONFEBASK. http://www.ieae. net/file.php/1/Estudios/Informe_Caliad_en_la_Gestion_en_PYMES/Informe_CalidadGestion. pdf (mayo 2011)

Camisón C., Gonzalez T. and Cruz S., 2007. Gestión de la Calidad. Madrid: Pearson Educación.

Cánovas F. and Pardavila B., 2004. La gestión de la calidad en los servicios sociales. Madrid: IMSERSO.

Caro F., 2001. Efectos de la Innovación y el Cambio en la Gestión y Organización de Empresas Informativas. Sevilla: Universidad de Sevilla.

Consejo Estatal de ONGs de Acción Social, 2006. Plan Estratégico del Tercer Sector. Madrid: Plataforma de ONG de Acción Social. http://www.plataformaong.org/upload/56/09/Plan_Estrategico_del_Tercer_Sector.pdf

EFQM, 2003. Modelo EFQM de Excelencia. Versión para el Sector Público y las Organizaciones del Voluntariado, Club Gestión de la Calidad.

Eisenhardt K.M., 1989. Building theories from case study research. Academy of Management Review, 14(4), 532-550.

Garau J., 2005. Guía para la gestión de la Calidad de los procesos de servicios sociales. Madrid: Ministerio de Trabajo y Asuntos Sociales. http://www.ongconcalidad.org/guiaprocesos.pdf

Grandzol J.R. and Gershon M., 1998. A survey instrument for standardizing TQM modeling research. International Journal of Quality Science, 3, 80-105.

Hamlin, B., Reidy M. and Stewart J., 1997. Changing the management culture in one part of the British Civil Service through visionary leadership and strategically led research-based OD interventions. Journal of Applied Management Studies, 6(2), 233-251.

Herrera Gómez M., Gallego Martínez D. and Páges Luis S., 2000. Una nueva realidad en los sistemas de bienestar: el Tercer Sector. En: Requena A.T. (ed.): Evaluación y calidad en las Organizaciones Públicas. Madrid: INAP, 113-141.

Ho D.C.K., Duffy V.G. and Shih H.M., 1999. An empirical analysis of effective TQM implementation in the Hong Kong electronics manufacturing industry. Human Factors and Ergonomics in Manufacturing, 9(1), 1-25.

INCONG, 2009. Norma ONG con Calidad (Tercera versión) http://www.icong.org/NormaONGconCalidad-v3.pdf

Juaneda Ayensa E., 2010. Retos de las organizaciones del tercer sector en el modelo de bienestar: implantación de sistemas de gestión de calidad total y compromiso organizativo. Tesis Doctoral. Logroño: Servicio de Publicaciones de la Universidad de La Rioja.

Lorenzo J.D., 2003. El cambio en las organizaciones: un modelo dinámico e integrador. Cádiz: Servicio de Publicaciones de la Universidad de Cádiz.

Martínez Caro L., Flores López E. and Martínez García J.A., 2010. Análisis de las necesidades del cliente y su satisfacción en la industria del mueble según las normas ISO 9000: un estudio de casos. Cuadernos de Gestión, 10(2), 99-116.

Sajardo A., Chaves R., 2006. Balance y tendencias en la investigación sobre el Tercer Sector no lucrativo. Especial referencia al caso español. CIRIEC-España, Revista de economía pública, social y cooperativa, (56), 87-116.

Salamon L. and Anheier H., 1998. Social origins of Civil Society: explaining the Nonprofit Sector cross-nationally. Voluntas: International Journal of Voluntary and Nonprofit Organizations, 9(3), 213-248.

Sánchez Aguado A., 2011. La calidad en la gestión de los Servicios Sociales Municipales. Algunas experiencias de INTRESS. TS nova: trabajo social y servicios sociales, (3), 49-54.

Shin D., Kalinowski J.G. and El-Enein G.A., 1998. Critical implementation issues in total quality management. SAM Advanced Management Journal, 63(1), 10-15. 
Yin R.K., 1994. Case study research: design and methods. California: Sage Publications Inc.

Yin R.K., 1998. The abridged version of case study research. En: Bickman L. and Rog D.J., eds. Handbook of applied social research methods. California: Sage Publications, Thousand Oaks, 229-259.

Zeitz G., Johannesson R. and Ritchie J.E., 1997. An employee survey measuring total quality management practices and culture. Group and Organization Management, (22), 414-444. 


\section{ANEXO 1}

Bloque de Preguntas 1. ¿Cuál es el punto de partida de la organización? Es decir, cómo define su misión y visión. Se pretende conocer el fin para el que se crea la organización y cómo se plantea su futuro.

Bloque de Preguntas 2. ¿Qué elementos del entorno actúan como fuentes de cambio? Una clave para poder alcanzar la visión es la adaptación y gestión de los factores del entorno. Esto exige la capacidad de análisis de la información externa.

Bloque de Preguntas 3. ¿Cuáles han sido los principales cambios realizados en la organización? El objetivo de esta pregunta es contrastar cómo se ha procedido en la gestión del cambio: priorización de riesgos, toma de decisiones, la implantación y los resultados obtenidos. También nos permite extrapolar posibles actuaciones futuras.

Bloque de Preguntas 4. ¿Cómo se lidera el cambio en la organización? En este apartado se recoge un principio clave de TQM, pretendiendo determinar la manera en que se establece la estrategia de la organización en consonancia con la misión y la visión; la comunicación a los demás stakeholders; y si se promueve explícitamente un sistema de valores.

Bloque de Preguntas 5. ¿Cómo se define la Estrategia de la organización? Este apartado se trata anteriormente en el Agente Liderazgo, por ello lo que nos interesa saber es su relación con herramientas utilizadas como autoevaluaciones, colaboraciones, indicadores etc.

Bloque de Preguntas 6. ¿Cómo se desarrolla el compromiso de las personas en la actividad de la organización? Consideraremos elementos como el trabajo en equipo, la medición de la satisfacción de los trabajadores/voluntarios, la transferencia de información, el sistema retributivo y su sistema de valoración del rendimiento; y planes de formación y gestión del conocimiento.

Bloque de Preguntas 7. ¿Cuáles son los principales grupos de interés para la organización? El desarrollo de este punto nos permite conocer las relaciones que se establecen con agentes externos a la organización, y la manera en que se favorece a la colaboración en busca de sinergias.

Bloque de Preguntas 8. ¿Existe una orientación hacia los procesos? Finalmente la última pregunta pretende contrastar si existe una orientación hacia los clientes y si interfieren a la hora de establecer el diseño de las actividades y la ejecución de éstas. 